ARTICLES

\title{
LA PLACE DE LA PSYCHOLOGIE DANS L'ORDRE DES SCIENCES
}

\author{
Fernando VIDAL
}

«Psychologie : piste l'homme et, close et figée, lui inflige une autopsie. »

Michel Leiris, Langage Tangage.

Résumé : L'histoire de la psychologie en tant que discipline autonome comporte non seulement des développements méthodologiques et institutionnels, mais aussi l'élaboration du concept même de psychologie et des représentations de sa place dans l'ordre des sciences. Si de telles représentations ne déterminent pas la constitution du champ professionnel ou la pratique concrète du psychologue, elles n'en expriment pas moins des idéaux épistémologiques et reflètent les changements qui s'opèrent au sein de la discipline. Nous donnerons d'abord une vue générale de la position de la psychologie au xvif et au xviff siecle, puis nous esquisserons l'histoire de sa place dans l'ordre des sciences de Destutt de Tracy à Jean Piaget. Après avoir été la science des sciences des classifications hiérarchiques, la psychologie s'intègre aux autres sciences dans des schémas moins linéaires, voire circulaires, sans pour autant toujours perdre la prééminence gnoséologique et pédagogique que lui attribuèrent en premier les * psychologues "du xvIf siècle.

L'histoire de la psychologie en tant que discipline autonome porte non seulement sur ses développements théoriques, méthodologiques et institutionnels, mais aussi sur l'élaboration du concept même de psychologie et sur les représentations de sa place parmi les autres domaines du savoir. Parmi de telles représentations, les classifications des sciences ont une fonction prééminente : elles impliquent une réflexion qui dépasse les problématiques propres à chaque discipline; elles s'inscrivent en général dans un projet de caractère encyclopédique, et sont souvent liées à la recherche d'une langue ou d'une logique qui seraient communes à toutes les formes de connaissance. Précisément parce qu'elles se construisent en stylisant des situations de fait et en poursuivant des idéaux métascientifiques, elles contribuent non seulement à répertorier, mais aussi à définir des champs de savoir. C'est ce qu'avait vu Francis Bacon (1986, p. 76) lorsqu'il disait que

Revue de synthèse : IV' S. N ${ }^{\text {os }}$ 3-4, juillet-décembre 1994. 
les divisions reçues des sciences ne conviennent qu'à la somme reçue des sciences et que «le globe intellectuel est semblable au terrestre: on y trouve à la fois des terres cultivées et des régions désertiques »!

En ce qui concerne la psychologie, les classifications des sciences ont une double importance. En premier lieu, du moment où la psychologie a cherché à s'instituer en science autonome, elle a dô montrer comment elle s'insérait dans l'ordre général des sciences. C'était là une des meilleures manières de se justifier elle-même. $O r$, une telle quête d'insertion donna lieu à des débats au sujet de la définition même de la psychologie, et ces débats se sont avérés cruciaux pour son destin. C'est pourquoi, en deuxième lieu, les classifications des sciences fournissent un point de vue privilégié sur l'histoire entière de la psychologie. Elles mettent à jour les racines conceptuelles de la discipline, reflètent son élévation au rang de science des sciences et son ultérieure intégration dans le système des connaissances.

Il serait sans doute possible d'écrire une histoire de la psychologie en partant de celle que nous esquisserons ici. Elle ne serait certes pas exhaustive, mais elle montrerait que l'institutionnalisation de la discipline et l'aspiration à la scientificité qui constitue un de ses grands thèmes existentiels n'ont pas annulé la question de ses frontières, mais en ont fait, au contraire, le problème central de son épistémologie 2 .

\section{ENTRE MÉTAPHYSIQUE ET PHYSIOLOGIE : DE LA RENAISSANCE AUXX IDÉOLOGUES}

Les classifications anciennes, médiévales et renaissantes, englobent sans exception des matières qu'on peut qualifier de « psychologiques ». La classification de Francis Bacon (1561-1626), pour prendre celle qui deviendra le point de départ obligé des essais ultérieurs en la matière, rapporte chaque

1. Pour plus de précisions sur les textes cités dans cet article, se reporter à la Bibliographie, p. 350-353. Spezac.I, 1973, est une bonne introduction au probleme de la classification des sciences. Voir Cofl.son, 1966, pour un panorama historique, et MCRaE, 1961, prour la période de Bacon à Karl. KéDrov, 1977-1980, est l'étude d'ensemble la plus complète (mais présente quelques omissions sumprenantes); elle comprend une classification originale inspirée du matérialisme dialectique. On peut aussi consulter MouHasseb, 1971-1972. Bien que lacunaire, le travail le plus utile du point de vue documentaire reste RıcHARDson, 1964, paru en 1901 ; il foumit une liste chronologique et bibliographique, parfois avec des résumés, de presque 150 systèmes de classifications des sciences de Platon au début du $x x^{e}$ siècle. Enfin, voir le $n^{0}$ spécial « La classification des sciences *, Revue de synthèse, t. cxv, $4^{c}$ s., $n^{\text {os }} 1-2$, janv-juin 1994.

2. Il existe une seule recherche publiée sur la place de la psychologie : Braun et Barigeau, 1984. Elle comprend une esquisse des principes des classifications de Bacon a Engels, un résumé de la position de la psychologie, et une présentation des idées de Piaget et de Kédrov (les deux premières parties semblent en grande partie puisées chez Kédrov). 
groupe de connaissances - l'histoire, la poésie et la philosophie - respectivement à la mémoire, à l'imagination et à la raison. Dans la philosophie, Bacon propose de créer une " science générale de la nature et de l'état de l'homme ", dont une partie porterait sur ses misères et ses prérogatives, l'autre sur l' « alliance » de l'âme et du corps; cette seconde partie renferme certains thèmes d'ordre psychologique. Néanmoins, c'est la religion qui s'occupe de l'âme rationnelle ou " souffle de vie "; une science naturelle que Bacon ne nomme pas traite de l'âme en tant que substance corporelle; et ce sont la logique et la morale qui abordent l'entendement, la raison, l'imagination, la mémoire, l'appétit et la volonté (Bacon, 1842, liv. 4, chap. 1 sur la science générale de l'homme, chap. 3 sur la science de l'âme hụmaine).

Le schéma baconien, comme ceux qui le précèdent, met en relief l'éparpillement de la matière psychologique, sans faire de son étude un domaine séparé. Un tel éparpillement ne dément nullement la place supérieure, au sein de la science de la nature, qu'Aristote (De anima 1402 a 1-9) attribuait à l'étude de l'âme en tant que principe des êtres animés. Savonarole (14521498), par exemple, compilant Aristote, Thomas d'Aquin et Albert le Grand, estimait que les traités de l'âme doivent précéder tous les autres dans la philosophie naturelle, en vertu de la plus grande "noblesse" de leur objet. L'intellect étant immatériel, il appartient au métaphysicien d'en disputer; le physicus " traite de l'âme intellective en tant qu'elle est unie au corps, et non pas en tant qu'elle est séparée " (Savonarole, 1989, p. 113$114)^{3}$. C'est la même division que Bacon adopte et que réitère, parmi bien d'autres textes, le plus grand manuel aristotélicien français de la Renaissance tardive, le Corps de philosophie de Scipion Du Pleix (1569-1661): l'étude de l'âme unie au corps appartient à la " physique ", celle de l'âme en tant que substance séparée, à la métaphysique ou « science surnaturelle " (Du Pleix, 1990, liv. 8; 1992, liv. 5).

La division entre une science de l'âme rationnelle et une science de l'âme corporelle est un lieu commun qui finit par intégrer le concept de psychologia. Dès la fin du $x^{e}{ }^{e}$ siècle, le mot désigne la doctrine de l'animus ou du mens (l'âme « séparée ", immortelle, immatérielle), non pas de l'anima (l'âme en tant que principe vital uni au corps). C'est pourquoi, au début, une Psychologia n'est pas la même chose qu'un traité De anima.

3. Voir le livre 1, "De la division des sciences ", et le livre 2, " De l'ordre et de la dignité de chaque science ". Le titre original est Apologeticus de ratione poetica artis; celui de certaines éditions, Opus perutile de divisione ordine ac utilitate omnium scientarum, dit mieux son projet; l'Apologeticus y est inséré dans un recueil intitulé Compendium totius philosophiae tam naturalis quam moralis; plusieurs livres de la philosophie naturelle sont consacrés à l'âme. Voir Savonarola, 1982-1988: Apologeticus dans vol. 1, Compendium dans vol. 2. 
Les différences s'estomperont vite. Au xviI siècle déjà, lexiques, encyclopédies, manuels et commentaires situent la psychologie dans la pneumatologie, doctrine des substances spirituelles dont les autres branches sont la théologie et l'angélographie, mais également au sein de l'anthropologie. dont l'autre partie est l'anatomie. Très tôt, l'unité du lexique a masqué la disparité des projets.

En 1630, Johann Heinrich Alsted (1588-1638), théologien commentateur de Lull et proche de Ramus par sa conception de la méthode, donne de la distinction qui nous occupe une formulation aussi éphémère que significative. Dans son Encyclopadia universa, l'un des ouvrages les plus diffusés dans l'Europe de son époque, il situe la pneumatologie dans la philosophie théorique, entre la métaphysique et la physique. Il lui rattache la psychologie, science de la nature et des facultés de l'âme rationnelle (Alsted, 1649 , t. 2, p. 96) :

\section{DELINEATHO PSYCHOLOGIAE}

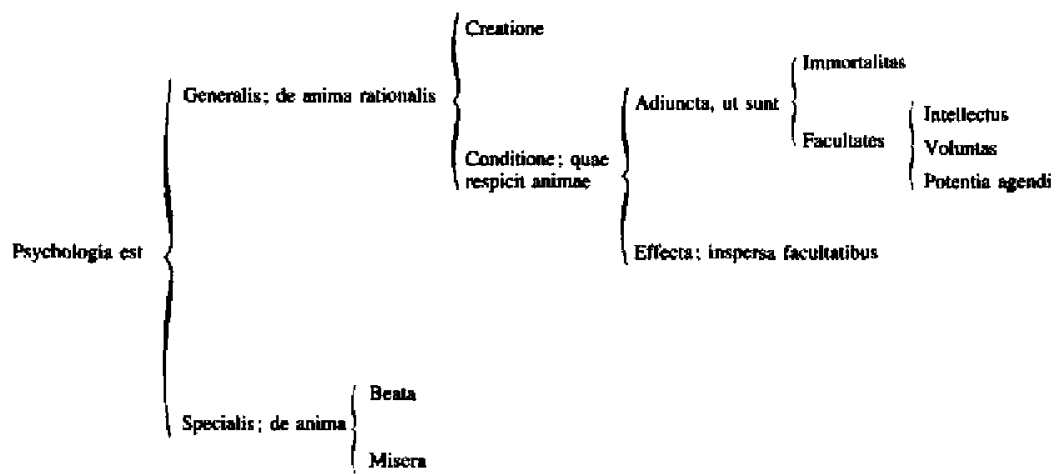

En fait, Alsted considère que la psychologia appartient non seulement à la métaphysique, mais aussi à la physique : mais lorsqu'il l'envisage comme science naturelle, il la nomme empsychologia ${ }^{4}$. Cet hapax dérive de l'adjectif empsuchos, qui qualifie le corps animé par la psuché. L'empsychologia est donc l'étude de l'âme en tant que principe animateur du corps, avec ses facultés vitales, végétatives et sensitives.

Le mot empsychologia ne durera pas. Alsted lui-même ne l'inscrit pas dans un schéma où il fait de l'étude des fonctions matérielles de l'âme l'une des divisions de la psychologia (ibid, p. 219). En étendant cette dernière à l'étude de l'anima sans lui faire perdre sa fonction première de science de l'animus, Alsted participe a la formation du double discours qui marquera dès lors le concept de psychologie.

4. Alsted, 1649, t. 2, Philosophia theoretica: liv. 12 (Pneumatica), $4^{e}$ partie (Psychologia); liv. 13 (Physica), 5 partie (Empsychologia et Phythologia). 
La présence de la psychologie dans les lexiques et les encyclopédies n'implique pas qu'elle se soit définitivement établie dans l'ordre des connaissances. Thomas Hobbes (1588-1679), par exemple, divise le savoir en connaissance des faits et connaissance de la consécution liant une affirmation à une autre; la première est pour lui absolue et donne lieu à l' " histoire »; la seconde est conditionnelle et donne lieu à la « science » ou "philosophie». Les livres de philosophie se divisent selon leur matière. Selon Hobbes (1971, chap. 9), les conséquences des qualités des animaux en général et de l'homme en particulier sont contenues dans des ouvrages d'optique, de musique, d'éthique, de poésie, de rhétorique, de logique; aucune science ne réunit en une sorte d'empsychologia les conséquences de l'ensemble de ces qualités.

À la fin du xvir siècle, dans un fragment d'après 1696, Gottfried Wilhelm Leibniz (1646-1716), qui connaît bien l'encyclopédie d'Alsted, définit la psychologie comme la science des monades, et la divise en doctrines de percipientibus in genere et de intelligentibus seu spiritibus (pneumatologie) ; contrairement aux partitions traditionnelles, la pneumatologie se trouve être une des branches de la psychologie (Couturat, 1966, p. 524529). Le sens que Leibniz donne à psychologia souligne le flottement sémantique du mot et le caractère largement non psychologique du concept originel.

Quant à John Locke (1632-1704), qui passe pour un des pères fondateurs de la psychologie, il est peu probable qu'il l'eât mentionnée s'il avait énuméré les sciences dans l'esquisse de classification placée à la fin de l'Essai sur l'entendement humain de 1690 . Locke $(1989$, liv. 4 , chap. 21) divise les sciences en philosophie naturelle, ethique et doctrine des signes. La philosophie naturelle vise la connaissance spéculative des choses, y compris des corps matériels et des substances spirituelles (Dieu, les anges et les esprits). Elle comprend donc la science de l'âme, mais non la psychologie proprement dite. En effet, la Psychologia à laquelle Locke aurait pu renvoyer défendait la doctrine de l'âme immatérielle et immortelle avec une argumentation qui constituait pour lui le type même de métaphysique à combattre ${ }^{5}$. Les connotations de psychologia feront que, pendant le xviII siècle, le projet d'une science empirique des facultés mentales ne prendra ce nom que lorsqu'il s'accompagnera d'une visée métaphysique et religieuse (Vidal, 1993).

Christian Wolff (1679-1754) fait de la psychologie à la fois une science des facultés, empirique, a posteriori, et un discours sur la nature de l'âme « rationnel », déductif, a priori. Charles Bonnet (1720-1793) aborde la psy-

5. Il s'agit de Psychologia : or, an Account of the Nature of the Rational Soul, par John Broughron, Londres, 1703 ; Locke, qui y est critiqué, réagit dans une lettre à Anthony Collins du 30 juin 1703. 
chologie comme une science d'inspiration sensualiste; il refuse de lui faire traiter la nature dernière de l'âme, mais la considère tout de même comme un condensé de sa métaphysique chrétienne. Pour l'un comme pour l'autre, la psychologie est intellectuellement et pédagogiquement première : Bonnet la situe à la base des études de philosophie; Wolff estime qu'elle fournit des principes au droit naturel, à la théologie naturelle, à la philosophie pratique et à la logique. La psychologie telle que la conçoivent Bonnet et Wolff est facilement acceptée par les chrétiens déclarés, disposés à mettre la science et la philosophie au service de la religion. Alors que l'Encyclopédie de Diderot et D'Alembert lui réserve un minuscule article wolffien, l'exclut du «Système figuré des connoissances humaines » et n'y renvoie jamais, l'Encyclopédie d'Yverdon, plus orthodoxe du point de vue religieux, consacre à la psychologie un article important, lui associe de nombreux termes, et l'érige en une science centrale, à laquelle sont reliées la métaphysique, la philosophie, la pneumatologie, l'anthropologie, la physiologie et l'économie animale.

Ceux qui se réclament de Locke et de la révolte contre la « scolastique » cherchent à rebâtir la logique et la philosophie sur des fondements en grande partie psychologiques. David Hume (1711-1776) le dira clairement : en expliquant les principes de la nature humaine et en fondant les sciences sur la « science de l'homme », il s'agit en fait de donner des bases nouvelles au système des sciences dans sa totalité (Hume, 1973, Introduction). Et le cour de cette science de l'homme, c'est la psychologie.

Si l'abbé de Condillac (1715-1780) refuse d'employer le mot « psychologie ", c'est seulement à cause de ses connotations métaphysiques; comme les encyclopédistes et autres philosophes avant la réfutation qu'en donne Kant dans la Critique de la raison pure, il conteste le principe même d'une «psychologie rationnelle». Une « première science», dit-il, aurait pour but de décomposer les idées, d'en déterminer l'origine et la génération; c'est cette science de l'analyse qu'il aurait nommée psychologie s'il avait connu «quelque bon ouvrage sous ce titre » (Condillac, 1947-1951, p. 299). Le refus de Condillac montre bien à quel point la prééminence qu'acquiert vers le milieu du xviI' siècle la science empirique de l'âme (des facultés cognitives en particulier) doit être distinguée de la formation d'une psychologie portant ce nom.

Avec la Révolution se font jour les enjeux politiques de la classification des connaissances. Il s'agit d'effacer les traces de l'Ancien Régime. Apparaissent alors nombre de nouveaux systèmes bibliographiques qui s'accordent pour faire descendre la théologie aux derniers rangs, voire la faire disparaître au profit de la métaphysique, mais qui, pour le reste, different totalement (Brunet, 1865, cols ix-xii). Destutt de Tracy (17541836), qui eut son mot à dire en matière de bibliographie, rejette le terme 
de psychologie parce qu'il veut dire «science de l'âme » et fait penser à « la recherche vague des causes premières ». Néanmoins, lorsqu'il se propose de ranger les connaissances dans l'ordre de leur dépendance les unes des autres, il place en tête l' " analyse de la pensée " qu'il nomme idéolo$g^{i} e^{6}$. Dès lors, la résistance au mot ne fait que trahir la transformation du concept. Et là où le mot se propage, le concept fait fortune; c'est le cas de la "psychologie empirique » dans l'Allemagne de la fin du xvil" siècle (Dessoir, 1964).

NATURPHILOSOPHIE, UTILITARISME, KANTISME :

HEGEL, BENTHAM, AMPÈRE

La place attribuée à la psychologie dans une classification dépend toujours d'une philosophie particulière; elle s'y réduit parfois entièrement. Ainsi de Hegel (1770-1831), pour qui la psychologie appartient à la philosophie de l'esprit. Celle-ci porte sur l'esprit subjectif (siège des phénomènes psychologiques élémentaires), l'esprit objectif (qui se manifeste dans les mours, les lois, la moralité, la religion et les sciences) et l'esprit absolu (siège de l'art, la religion et la philosophie). La psychologie est la troisième partie de l'étude de l'esprit subjectif, qui englobe également l'anthropologie et la phénoménologie de l'esprit. L'anthropologie porte sur l'esprit en soi ou immédiat, autrement dit sur l'âme naturelle, sur l'ầme sensible et sur l'âme réelle (cette dernière est l'âme se manifestant et agissant par le moyen du corps). La phénoménologie de l'esprit traite de l'esprit pour soi ou médiatisé (la conscience, évoluant de la sensation à la raison). La psychologie, enfin, étudie l'esprit se déterminant soi-même, comme sujet pour soi; elle comprend la description de l'esprit théorique (des fonctions telles que l'imagination, la mémoire et la pensée) et de l'esprit pratique (les tendances, les passions et les désirs), dont l'union produit l'esprit libre. La place de la psychologie chez Hegel (1952) est déterminée par le rythme triadique de sa pensée et n'échappe pas aux obscurités qui lui sont propres. Dans un fragment de 1798-1799, son contemporain Novalis (1772-1801) notera plus poétiquement que psychologie et physiologie pourraient ne faire qu'une seule science, dont l'âme serait le principe : «La physiologie en général serait la psychologie du monde - et nature et âme ne feraient également qu'un » (Novalis, 1966, p. 271).

Tout autre, mais non moins' centrale, est la place de la psychologie du point de vue utilitariste. Conformément à son principe d'utilité, Jeremy

6. Destutt de Tracy, Mémoire sur la faculté de penser, 1798, in 1992, p. 71, et Sur un système méthodique de bibliographie, 1797, in 1992. 
Bentham (1748-1832) subordonne toutes les sciences à l'« eudémonique », science générale destinée à maximiser le well-being de l'humanité. Bentham considère que la doctrine du bien-être est la véritable ontologie, et la divise en une science «coenoscopique » de ce qui est commun à tous les êtres et une science «idioscopique » de ce qui est propre à chaque classe d'êtres. L'ontologie idioscopique peut être «somatoscopique " (sciences mathématiques et naturelles) ou "pneumatoscopique » (pneumatologie); de bifurcation en bifurcation, cette dernière comprend la logique, la grammaire, la rhétorique, l'esthétique et l'éthique (dont les dichotomies successives mènent jusqu'à la politique et à la législation). Bentham ne fait pas figurer la psychologie dans son tableau des sciences. Ailleurs, pourtant, il l'identifie à la pneumatologie et la décrit comme l'une des deux branches de l'ontologie, l'autre étant la somatologie; de ces deux branches, dit-il, l'eudémonique dérive sa connaissance des phénomènes de la vie humaine relatifs a son but ${ }^{7}$. La psychologie, bien que ne figurant pas graphiquement au sommet du savoir, possède une préminence de fait, puisqu'elle fonde les techniques de gestion sociale destinées à susciter le bien-être général. Ce qui ne saurait surprendre vu l'importance dans la pensée de Bentham des considérations psychologiques, sur les motifs de l'action en particulier.

Chez Bentham, la psychologisation des concepts fondateurs touchait au domaine du gouvernement. L'ouvre d'Ampere illustre comment elle se poursuit en théorie de la connaissance. Outre ses travaux de physique et de mathématique, André-Marie Ampère (1775-1836) laisse (inachevé) un essai de «classification naturelle de toutes les connaissances humaines ». Sous l'influence de Kant, Ampère stipule l'existence de deux mondes : un monde phénoménal, que nous connaissons par nos impressions et où, par exemple, « les couleurs sont sur les objets »; et un monde nouménal hypothétique où, en revanche, " les couleurs sont des sensations excitées dans l'être sentant par certains rayons et qui n'existent qu'en cet être ". Le monde nouménal des astronomes et des physiciens est fait d'intuitions de relations (telles la durée, le mouvement ou le nombre) indépendantes des impressions sensorielles. Ces considérations fondent chez Ampère la taxinomie des phénomènes psychologiques, dont il s'est occupé dans des lettres à Maine de Biran et dans ses cours du Collège de France, ainsi que la classification des sciences (Bréhier, 1986, p. 561-567).

Ampère divise les sciences en deux "règnes", celui des sciences du monde ou cosmologiques et celui des sciences de la pensée ou « noologiques ». L'ensemble se constitue par bifurcations successives. Les

7. Chrestomathia, 1816, Table V, in BentHaM, 1962 ; Appendix IV : Essay on Nomenclature and Classification », ibid., p. 84; * Fragment on Ontology * (manuscrits de 1813, 1814 et 1821), ibid., p. 195; « Logic * (manuscrits datant d'entre 1811 et 1831), ibid, p. 289. 
sciences noologiques sont philosophiques ou dialegmatiques (celles-ci étudient les signes servant à transmettre les idées, les sentiments, les passions). Les sciences philosophiques se divisent en morales et en philosophiques proprement dites; ces dernières sont la métaphysique et la psychologie. La psychologie étudie ce que l'on peut connaître « relativement à la pensée considérée en elle-même » (Ampère, 1934/1843, $2^{c}$ partie, p. 15).

Ampère classe les sciences selon quatre points de vue, qu'il appelle autoptique, cryptoristique, troponomique et cryptologique, portant respectivement sur la description des phénomènes, sur leur explication causale en termes de noumènes (qu'Ampère identifie à des entités théoriques telles que l'atome), sur la formulation des lois gouvernant les relations entre phénomènes, et sur l'explication de ces lois à partir de relations entre noumènes. À l'intérieur des sciences philosophiques, la psychologie représente le point de vue autoptique. Comme toutes les sciences, elle se divise à son tour selon les quatre points de vue : la psychographie décrit les « faits intellectuels »; la logique cherche à comprendre « les circonstances et les motifs » qui déterminent les jugements; la méthodologie porte sur l'agencement des connaissances (y compris les méthodes de classification, d'induction et d'enseignement); et l'idéogénie s'interroge sur « les sources et l'origine de nos idées » (ibid., p. 9-18).

Bien que la psychologie chez Ampère ne soit pas une propédeutique générale ni une science des sciences, elle est dans la pratique le fondement de son épistémologie. Car, au-delà de la distinction philosophique des mondes phénoménal et nouménal, ce sont les voies par lesquelles l'esprit connaît ces mondes qui déterminent la systématique des sciences; et c'est pourquoi Ampère considère que sa classification est « naturelle ».

\section{LA PSYCHOLOGIE AUX ORIGINES DU POSITIVISME}

Au $\mathrm{XIX}^{\mathrm{e}}$ siècle, la conceptualisation de la psychologie devient un des enjeux majeurs du débat philosophique; la place qu'on attribue à la psychologie dans le système des sciences s'érige en emblème des positions qui s'affrontent sur le terrain de l'épistémologie. Ainsi que le fait ressortir le positivisme, il y a là une conséquence de la croyance en la possibilité de fonder un nouvel ordre cognitif et social sur l'étude scientifique des fonctions mentales.

Le comte de Saint-Simon (1760-1825) imagine que, comme les philosophes du XVII ${ }^{e}$ siècle « ont fait une encyclopédie pour renverser le système théologique et féodal », ceux du $\mathrm{XIX}^{e}$ doivent en faire une nouvelle « pour 
constituer le système industriel et scientifique ${ }^{8}$. Il y contribue par un « arbre encyclopédique »: sur le feuillage près de la cime s'inscrivent les «sensations primitives» et l'«établissement des premiers signes de conventions »; sur le tronc principal (science générale), dont le tiers supérieur se perd dans le feuillage, s'affichent, de haut en bas, différentes époques : idolâtrie (époque des arts et des métiers), polythéisme (époque des beaux-arts), déisme (époque des sciences morales et politiques) et physicisme (époque des sciences mathématiques et physiques). Deux branches mères (sciences mathématiques et sciences physiques), poussent vers le haut presque verticalement, à partir du bas du tronc, et forment des branches disposées plus horizontalement. La branche des sciences physiques donne naissance aux branches de l'histoire naturelle, de la physique céleste, de la chimie, ainsi qu'aux deux branches parallèles de la science de l'homme et de la physiologie. Celles-ci s'élèvent vers le feuillage et sont reliées par une banderole : Rapports du physique et du moral de l'homme de Cabanis ${ }^{9}$; choix tout naturel, dans la mesure où, pour Saint-Simon, les physiologistes doivent chasser les philosophes, les moralistes et les métaphysiciens comme les astronomes et les chimistes ont autrefois chassé les astrologues et les alchimistes ${ }^{10}$.

La place que Saint-Simon donne à l'ouvrage de Cabanis laisse entrevoir celle que la psychologie aurait pu avoir dans l' « échelle encyclopédique » de son disciple Auguste Comte (1798-1857). Comte organise les sciences positives fondamentales selon un ordre qu'il estime être de complexité croissante et de généralité décroissante : mathématique, astronomie, physique, chimie, biologie et sociologie. (Ces sciences sont fondamentales dans la mesure où elles fournissent les bases théoriques des sciences particulières et descriptives : la chimie, par exemple, est la base de la minéralogie.) Cet ordre est moins historique que « dogmatique "; il represente « le système des idées tel qu'il pourrait être conçu aujourd'hui par un seul esprit, qui, placé au point de vue convenable et pourvu des connaissances suffisantes, s'occuperait à refaire la science dans son ensemble » (in Laubier, 1974, p. 38). Il est aussi un ordre didactique - ce qui fera dire à Friedrich Engels (1820-1895) qu'il conduit « à la folie de l'enseignement intégral », et à Charles Renouvier (1815-1903), qu'il suppose " terminée l’ère de l'exploration et de la recherche " et qu'il n'est « qu'une fiction et un appel à la foi, en vue de mettre fín à toute critique et de donner des chaînes

8. « Sur la nécessité de faire une nouvelle Encyclopédie pour préparer la réorganisation sociale », Opinions littéraires, philosophiques et industrielles, 1825, Cinquième opinion, in SAINT-SimoN, 1973, vol. 3, p. 259.

9. Dépliant placé à la fin du tome 1 de l'Introduction aux travaux scientifiques du dixneuvième siècle, 1807-1808, in SAINT-SimON, 1973, vol. 1.

10. Lettres d'un habitant de Genève d ses contemporains, 1802, in SAnNT-Smon, 1973, vol. 1, p. 24 . 
à l'esprit humain $\$$ (Engels, 1968, p. 254-255; Renouvier, 1912, p. 358). De cette échelle que Comte expose dans la $2^{\mathrm{e}}$ leçon du Cours de philosophie positive (1830-1842), la psychologie est absente.

Reprenant dans la $1^{\text {re }}$ leçon du Cours certaines idées de sa jeunesse, Comte déclare l'esprit incapable de s'observer soi-même. Dans la $45^{\mathrm{e}}$ leçon, il explique qu'une psychologie positive ne peut étudier que les productions de l'esprit humain et les fondements organiques des phénomènes psychiques, combinant ainsi la sociologie et la physiologie cérébrale. Comme le montre Annie Petit dans ce volume (p. 393-416), entre le Cours de philosophie positive et le Système de politique positive (18511854), la science de l'esprit (ou « théorie cérébrale positive ») devient plus nettement sociologique. La nature et la marche des facultés, selon Comte, sont les mêmes chez l'individu et dans l'espèce; mais comme seul ce dernier cas est « assez réel et assez développé pour les caractériser », le principe de la théorie cérébrale devient : «L'inspiration sociologique contrôlée par l'appreciation zoologique » (in Laubier, 1974, p. 133).

John Stuart Mill (1806-1873) ironise sur la préférence de Comte pour la phrénologie, et assure la possibilité de l'introspection et d'une science proprement psychologique (Mill, 1898, p. $65-66 ; 1897$, chap. $4, \S 2$ ). À I'opposé, dans son essai « De quelques points de physiologie psychique » (1860), Émile Littré (1801-1881) prétend réduire la psychologie à la psychophysiologie (in Littré, 1876). Herbert Spencer décèle une contradiction entre les prémisses du système comtien, selon lesquelles toute connaissance est relative et provient de l'expérience, et le refus de la seule science qui permettrait à Comte de les démontrer ${ }^{11}$. Tous semblent oublier que, comme le soulignera Lévy-Bruhl (1900, chap. 5), Comte s'oppose à la méthode qui lui semble commune aux Idéologues, aux éclectiques français et aux philosophes écossais du common sense, et non au principe d'une science positive de l'esprit.

Certes, chez Comte, la place systématique de la psychologie reste tue. Néanmoins, $l^{+}$attention intense et soutenue que le philosophe portait aux questions psychologiques et la part qu'il leur attribuait dans le plan de sa « morale théorique » permettent d'imaginer l'importance qu'il aurait donnée explicitement à la psychologie s'il avait vécu au-delà de sa Politique positive ${ }^{12}$. Le problème de fond, dont l'apparente négation comtienne

11. "Pourquoi je me sépare d'Auguste Comte", 1864, in SPencer, 1909.

12. Il suffit d'examiner la "Classification positive des dix-huit fonctions intérieures du cerveau ou Tableau systématique de l'âme ", in Catéchisme positiviste, $1852,8^{\mathrm{e}}$ entretien, pour entrevoir la place centrale de la psychologie dans le systeme comtien. Edmond Goblot (1858-1935) et Adrien Naville (1845-1930) tenteront de dépasser le refus comtien, le premier en mettant l'accent sur l'unité de la psychologie et de la biologie et sur leur continuité avec la sociologie, le second en insistant sur l'interdépendance de la biologie, la psychologie et la sociologie. Voir Gohlot, 1898; NAYILLE, 1887, 1920. 
précise les termes, n'est pas de savoir si la psychologie a droit à une place parmi les sciences, mais de définir cette place eu égard à l'histoire des sciences, à la continuité et à l'interdépendance des phénomènes, et à la diversité des méthodes du savoir. Peut-on, en effet, établir des limites réelles (plutôt que nominales), alors que les objets à classer semblent s'organiser en séries continues?

\section{ENCHEVETREMENT ET CONTINUITÉ : COURNOT ET WHEWELL}

En 1851, année où commence à paraître le Système de politique positive, Antoine-Augustin Cournot (1801-1877) publie l'un des grands ouvrages épistémologiques du $\mathrm{XIX}^{\mathrm{e}}$ siècle, l'Essai sur les fondements de nos connaissances, qui traite en partie de "la coordination des connaissances humaines " et des "caractères scientifiques de la psychologie et de son rang parmi les sciences" (Cournot, 1975, chap. 22 et 23).

Après avoir critiqué l'abus de la méthode de la bifurcation chez Bentham et chez Ampère, ainsi que le caractère linéaire de leurs classifications, Cournot construit un tableau à double entrée. Il définit cinq "étages 》 ou types de sciences, conformes au schéma d'Ampère et disposés, comme chez Comte, selon un ordre de complexité croissante. Il s'agit des sciences mathématiques, physiques et cosmologiques, biologiques, noologiques et symboliques, et politiques et historiques. Ces cinq groupes se combinent avec trois « séries». À la série théorique appartiennent les sciences «dont le propre est de relier en systèmes des vérités éternelles ou des lois permanentes de la nature ». La série cosmologique englobe les sciences " qui portent sur un enchaînement de faits qui se sont produits successivement les uns les autres, et qu'on s'explique les uns par les autres, en remontant jusqu'à des faits originels qu'il faut admettre sans explication ». La série technique comprend les sciences appliquées (ibid., p. 408-409).

La psychologie empirique se situe à la frontière des sciences biologiques et noologiques. Les premières, explique Cournot, supposent les sciences physiques et cosmologiques, et conduisent, "par l'histoire naturelle de l'homme et par la psychologie empirique (étroitement unie à la physiologie) ", jusqu'à la limite des sciences de l'entendement et de la nature morale de l'homme; le passage se fait par des «transitions continues » allant « de l'étude des fonctions du système nerveux et de la sensibilité animale à l'étude des facultés supérieures de l'intelligence ». Il n'y a donc pas de «distinctions tranchées " dans la série des phénomènes psychologiques de la physiologie à la logique (ibid., p. 410-412). Les sciences biologiques et noologiques ensemble forment la série théorique suivante : anatomie, embryologie, tératologie, physiologie, phrénologie, physiognomonie, psy- 
chologie empirique [demière science biologique], idéologie, logique, esthétique, theologie naturelle, éthique (ibid., p. 408-409).

Contre Comte, Cournot (1986, p. 112) estime que la « psychologie superieure de l'homme " suppose la sociologie, autant que la sociologie suppose la biologie de l'individu; la continuité de la série implique l' " enchevêtrement " des phénomènes plutôt que la "superposition " des sciences. En vertu du postulat de la continuité des phénomènes, il ne donne, ni à la psychologie ni à aucune autre science, une position de prééminence historique ou épistémologique. Il en va de même chez William Whewell (17941866), auteur d'une des premières histoires générales des sciences.

Whewell (1858, chap. 9) explique qu'une classification des sciences doit se fonder sur les « idées » ou « conceptions » dont relèvent les principes de chaque science. Les idées d'une science conduisent graduellement à celles d'une autre; au seuil de la physiologie, par exemple, se trouvent les idées de vie, de sensation, de volonté, au sujet desquelles le philosophe déclare :

« It is plain that the pursuit of such conceptions and their consequences would lead us to the sciences (if we are allowed to call them sciences) which contemplate not only animal, but human principles of action, to Anthropology and Psychology " (ibid., p. 138).

Le tableau systématique de Whewell (ibid., p. 140) comprend une colonne pour les idées fondamentales, une autre pour les sciences et une troisième pour l'ordre encyclopédique. L'ensemble commence par l'espace (idée), la géométrie (science) et les sciences mathématiques pures (classe que la géométrie partage avec l'arithmétique, l'algèbre et le calcul différentiel). À la suite des sciences physiques et des sciences classificatoires (taxinomie et anatomie comparée), le système s'achève par les idées d'irritabilité, d'organisation et de cause finale (propres à la biologie), d'instinct, d'émotion et de pensée (psychologie), de causalité historique (diverses sciences) et de cause première (théologie naturelle). Comme chez Cournot, c'est en demière analyse la continuité des phénomènes (dont dériveraient par induction les idées fondamentales) qui donne à la psychologie sa place parmi les sciences. Qu'un fait psychologique se trouve en dehors de la série, et la psychologie tout entière sortira du système.

LA PSYCHOLOGIE, SCIENCE HORS PAIR : SPENCER ET BAIN

Dans un essai de 1854 sur la genèse de la science, Herbert Spencer (1820-1903) dit ne pas vouloir ordonner les sciences en une série; aucune 
série, selon lui, ne peut représenter les relations logiques ou historiques entre les sciences; et les classifications existantes se trompent en ce qui concerne et la logique et l'histoire (celle de Comte en particulier lui semble confondre l'ordre dogmatique et l'ordre historique). La vision de Spencer (1854) est axée sur la latéralité des relations et gouvernée par le souci de ne pas confondre l'abstrait et le général.

Dix ans plus tard, dans La Classification des sciences, Spencer explique que les sciences dites abstraites (logique, mathématiques) s'occupent de relations indépendamment des choses entre lesquelles existent ces relations. Les sciences abstraites-concrètes procèdent par abstraction à partir de phénomènes concrets (par exemple, la mécanique, la physique et la chimie aboutissent à des vérités qui ne portent sur aucun objet particulier, mais sur la matière et le mouvement en général). Finalement, les sciences concrètes visent non pas l'« explication analytique des phénomènes » (comme les sciences abstraites-concrètes), mais leur "interprétation synthétique »; elles cherchent non pas à abstraire et à généraliser les composants des phénomènes, mais à expliquer chaque phénomène comme le résultat de ces composants. Dans un « Post-scriptum, en réponse aux critiques », Spencer comparera la distinction entre l'abstrait et le concret à celle entre la fiction et la biographie, séparées selon lui par un abîme infranchissable. Par conséquent, les trois groupes des sciences ne forment pas une série. Chaque groupe fournit la matière du suivant; mais les faits établis par chacun n'aident pas à résoudre les questions propres aux autres. Il n'y a pas de filiation, ni historique, ni généalogique, ni logique, mais synchronie. (Les deux textes se trouvent dans Spencer, 1909.)

En revanche, selon Spencer, il y a série dans l'ordre des phénomènes dont s'occupe la science concrète; cette série commence par la manière dont les lois de la redistribution de la matière et du mouvement affectent les interactions des molécules, et aboutit à la production de phénomènes organiques sur la terre. La psychologie et la physiologie étudient la dimension fonctionnelle de ces phénomènes, respectivement dans leurs relations internes et externes (voir Spencer, 1909, Tableau III).

L'ordre spencérien des sciences est conforme à la célèbre définition des Premiers principes $(1894, \S 145)$ selon laquelle

«L'évolution est une intégration de matière accompagnée d'une dissipation de mouvement, pendant laquelle la matière passe d'une homogénéité indéfinie, incohérente, à une hétérogénéité définie, cohérente, et pendant laquelle aussi le mouvement retenu subit une transformation analogue. "

L'évolution ainsi conçue détermine la place de la psychologie parmi les sciences. C'est la raison pour laquelle Renouvier $(1912$, p. 185) reprochait a Spencer de trahir ses propres principes, en restituant au sein de sa clas- 
sification l'unité réductrice qu'il avait paru vouloir éviter. De l'astronomie à la sociologie, en passant par la géologie, la biologie et la psychologie, les sciences s'occupent d' « agrégats » de plus en plus spéciaux et hétérogènes évoluant les uns à partir des autres par gradations insensibles, et formant un ensemble de phénomènes interdépendants.

La psychologie, d'après Spencer, est une des sciences concrètes et possède des frontières purement conventionnelles avec la biologie et la sociologie. Pourtant, dans les Principes de psychologie (1874, \& 53-56), le philosophe affirme qu'elle est absolument unique, indépendante et même contraire à toutes les autres sciences. Sous l'angle de la subjectivité, la psychologie étudie la conscience, c'est-à-dire, selon Spencer, une entité à part, différente par sa nature des entités dont s'occupent les autres sciences; or, la «psychologie objective " ne pourrait pas exister sans la «psychologie subjective ». Alors que les sciences traitent des connexions entre phénomènes internes ou entre phénomènes externes à l'organisme, la psychologie porte sur la connexion entre ces deux connexions. Son objet procède de la même évolution qui détermine la continuité des sciences. Cependant, dans la mesure où la psychologie permet de décrire et d'expliquer les processus par lesquels on connaît et on élabore les sciences, elle est le fondement de tout discours métascientifique.

Le philosophe et psychologue écossais Alexander Bain (1818-1903), fondateur de la revue $M i n d$, consacre la plus grande partie de son résumé des classifications des sciences à critiquer Spencer (Bain, 1894, Appendice A). Pour lui, les sciences ne peuvent être qu'abstraites ou concrètes; les premières précèdent les secondes; et cette disposition correspond à l'ordre historique et pédagogique suivant : logique, mathématique, physique mécanique, physique moléculaire, chimie, biologie et psychologie. Bain considère que les relations entre les sciences vont dans tous les sens, non seulement des concrètes vers les abstraites. Il estime aussi que les sciences mêlent le concret et l'abstrait : la psychologie décrit et classifie; mais en plus elle décompose l'esprit en facteurs et fonctions liés entre eux (il s'agit pour Bain du sentiment, de la volonté et de la pensée).

Par-delà leur désaccord quant à la systématique des sciences, Spencer et Bain reconnaissent la singularité de la psychologie. Pour Bain, si la psychologie occupe la dernière place dans le développement des sciences, c'est parce que l'esprit est une entité extrêmement complexe, et que, malgré les liens du psychique et du physiologique, la conscience subjective reste un phénomène sui generis absolument unique et irréductible (ibid., p. 31-43). En vertu du postulat de la continuité des phénomènes, toutes les sciences appartiennent au système du savoir; en devenant l'exception qui confirme la règle, la psychologie voit renforcée sa position de science fondatrice. 
EMPIRISME ET PRAGMATISME : PEARSON ET PEIRCE

En 1892 paraît La Grammaire de la science, de Karl Pearson (18571936), mathématicien, statisticien et biométricien, promoteur de l'eugénisme et philosophe. Sa conception de la science le rapproche de l'épistémologie empiriste, antimétaphysique et nominaliste d'Ernst Mach. Pour Pearson (1900, p. 504), * the object of science is to describe in conceptual shorthand the routine of our past experience, with a view of predicting our future experience $»$. Cette " routine » est celle des sensations, conçue dans une optique associationniste. Dès lors, la science peut être ramenée à l'association, et les lois scientifiques ne sont que des formules résumant et réunissant des phénomènes connus au moyen de la perception. Les sciences elles-mêmes sont dépourvues de frontières bien définies; elles évoluent et sont interdépendantes: les organismes qu'étudie le microbiologiste, par exemple, s'avêreront très importants pour certains aspects de la médecine qui auront à leur tour un grand impact sur la sociologie. Il est donc impossible, pour Pearson, d'arriver a un système des sciences complet et cohérent.

Pearson divise les sciences en abstraites et concrètes, suivant qu'elles portent sur les modalités ou sur les contenus de la perception. Les sciences concrètes des phénomènes organiques traitent soit de phénomènes historiques, non répétitifs (l'évolution des espèces, y compris de l'organisme, des facultes mentales et des institutions sociales de l'homme), soit de phénomènes biologiques. La partie de la biologie qui étudie les fonctions et les conduites peut porter sur des faits physiques (physiologie) ou sur des faits mentaux (psychologie). La psychologie générale étudie par exemple la théorie des instincts; la psychologie humaine peut se centrer soit sur l'individu, soit sur le groupe (ibid., p. 526). En plaçant la psychologie au sein de la biologie, Pearson indique l'importance pour sa classification de la croyance en la continuité du physique et du mental. Dans ce sens, la psychologie n'occupe pas une situation privilégiée.

Néanmoins, c'est de la psychologie que dérivent la sociologie et les autres sciences sociales (telles que l'économie et la jurisprudence). À cette place première au sein des sciences humaines s'ajoute la fonction de la psychologie en tant que fondement ultime de l'épistémologie. En effet, la classification de Pearson découle de son empirisme, et celui-ci repose sur une psychologie. D'après les critères du philosophe lui-même, seule la psy- 
chologie est capable de justifier la conception de la science comme grammaire.

Chez Spencer et Bain, et moins explicitement chez Pearson, la psychologie est à la fois science et métascience. Nul équivoque, en revanche, dans les systématiques qui ressortissent à des principes logiques ou autrement non psychologiques.

Dans sa classification des sciences ${ }^{13}$, Charles Sanders Peirce (18391914), le fondateur du pragmatisme, se propose de suivre les affinités des sciences entre elles, en accord avec l'idée de Comte, « that one science depends upon another for fundamental principles, but does not furnish such principles to that other » (1.180). Il existe pour lui deux sortes de sciences : theoriques et pratiques. La première comprend les sciences of discovery (les mathématiques, la philosophie et l'idioscopie dans le sens de Bentham) et les sciences of review (prenant forme dans des travaux tels que la Philosophie positive de Comte ou Synthetic philosophy de Spencer, censés aboutir à une philosophie de la science).

L'idioscopie se divise en sciences physiques (physiognosie) et psychiques (psychognosie), comprenant chacune des branches nomologiques, classificatoires et descriptives. Les domaines nomologiques et classificatoires de la psychognosie englobent diverses disciplines psychologiques: les spécialités introspective, expérimentale, physiologique et enfantine forment la psychologie proprement dite (nomological psychics); les psychologies individuelle, pathologique, animale et des races font partie de la psychognosie classificatoire.

Appliquant la norme de la dépendance hiérarchique, Peirce situe au sommet la mathématique (dont on trouve des applications dans toutes les sciences) et ensuite la philosophie (que chaque science particulière doit prendre en considération). Les sciences idioscopiques, dit-il, dépendent largement de la philosophie; par exemple, dans la mesure où la psychognosie n'accède pas directement au psychique mais doit l'induire d'états corporels (1.250), elle est redevable à la logique. Mais même si la psychologie repose en partie sur la physiognosie (1.253-255), il n'y a pas chevauchement: "Very little psychology is needed by the biologist; and no very deep biology by the psychologist " (1.265). En somme, la place de la psychologie dans le système de Peirce obéit à l'opposition du philosophe au psychologisme tant en théorie de la logique qu'en épistémologie.

13. "An Outline Classification of the Sciences " (1903) et "A Detailed Classification of the Sciences " (1902), respectivement chap. 1 et 2 de HarTSHORNE et Weiss, 1960, vol. 1 . Les différences entre les déux textes sont négligeables pour notre propos. Sur la place de la logique, voir en plus * Why Study Logic? (1902), § 1, chap. 3 de HaRTSHORNE et WeIss, 1960, vol. 2. Nous suivons la manière conventionnelle de citer les Collected Papers de Perrce, en donnant le numéro du volume suivi de celui du paragraphe. 
Dès la dernière décennie $\mathrm{du} \mathrm{XIX}^{\mathrm{e}}$ siècle, le débat que suscitent les idées de Wilhelm Wundt (1832-1920) sur la nature et la place de la psychologie parmi les sciences s'avère décisif pour la constitution de la psychologie comme discipline autonome (Danziger, 1979; Leary, 1979; Bringmann et Tweney, 1980). Wundt soutenait que la psychologie n'est pas une science naturelle, mais une Geisteswissenschaft (science de l'esprit ou science humaine). La psychologie ne se rapproche des sciences naturelles que par l'usage qu'elle fait de l'expérimentation. Néanmoins, cette méthode se limite à l'étude des processus psychologiques élémentaires; les processus complexes doivent être abordés par des méthodes non expérimentales, sociales et historiques. Dans la perspective de Wundt, l'existence même de la psychologie dépendait de l'acceptation d'une causalité psychique irréductible à la causalité physique, et supposait la légitimité de différentes sortes d'explication scientifique.

Wundt voyait dans la psychologie une science première du point de vue gnoséologique. D'abord, les sciences naturelles construisent leur matière à partir d'expériences psychologiques immédiates. Deuxièmement, la psychologie est le fondement de la théorie de la connaissance, de la logique et de la morale. Finalement, les autres sciences de l'esprit se basent sur des présuppositions psychologiques. Ainsi, bien que la psychologie ne fît que commencer à se détacher de la philosophie et à devenir une des « sciences individuelles (Wundt, 1906, p. 77), Wundt la situait au sommet des sciences humaines. En tant que théorie générale des processus spirituels, elle embrasse et sert de base à toutes les autres sciences qui traitent des productions de l'esprit (Wundt, 1889, p. 44-45).

Wundt divise les sciences en formelles (mathématiques pures) et " réelles (matérielles, empiriques); leurs applications pratiques ne se prêtent à aucune ordonnance logique. Les reale Wissenschaften comprennent les sciences naturelles et les sciences de l'esprit. Elles sont "phénoménologiques s lorsqu'elles étudient des processus (c'est le cas de la psychologie), « systématiques » lorsqu'elles étudient les productions de la nature ou de l'esprit (ainsi du droit et de l'économie), et "génétiques " lorsque, comme l'histoire, elles étudient la formation et le développement de ces productions (Wundt, 1906, $\$ 7 ; 1907,1^{\text {re }}$ partie, chap. 3, \$2). Envisagées historiquement, les sciences systématiques atteignent les premières un certain niveau d'élaboration, puis viennent les sciences génétiques et, enfin, les sciences phénoménologiques (Wundt, 1906, p. 76-77; cf. Wundt, 1907, p. 99). 
Dans un tel schéma, la psychologie fait figure đe médiatrice entre les sciences de la nature et les sciences de l'esprit: par ses méthodes, puisqu'elle comprend l'expérimentation caractéristique des sciences naturelles; par la signification purement méthodologique de l'idée de «parallelisme psychophysique ". Il n'y a pas selon Wundt de dualisme corpsesprit : la psychologie traite des processus propres à l'expérience interne du sujet, les sciences naturelles, des processus appartenant à des objets extérieurs au sujet qui les étudie.

L'agencement des sciences humaines fait ressortir la préeminence épistémologique de la psychologie (Wundt, 1889, p. 47). La première des sciences humaines phénoménologiques est la science des processus spirituels dans la conscience humaine (psychologie individuelle). Elle est suivie des sciences de ces processus envisagés dans des conditions particulières (psychologie animale, Völkerpsychologie, psychophysique, anthropologie, ethnologie). Ces différentes branches de la psychologie sont suivies d'aborit des sciences humaines systématiques (comprenant la philologie, l'éeonomie, les sciences politiques, la théorie du droit, la théologie systématiqué, la théorie des arts et la méthodologie des sciences), ensuite des sciences humaines historiques (depuis la biographie et l'histoire universelle jušiqu'à l'histoire de chaque classe de productions mentales, telle que l'histoire de l'art et l'histoire des sciences).

C'est Oswald Külpe (1862-1915) qui, le premier, mit en question la position de Wundt. Rejetant l'idée de causalité psychique, il affirmait que les phénomènes mentaux dépendaient de processus corporels, et déclarait que tous les sujets de la psychologie pouvaient être abordés expérimentalement. Le débat entre Wundt et Külpe oppose deux conceptions de la science, l'une supposant que les concepts de chaque science sont irréductibles à ceux d'une autre science, fût-ellè plus générale et plus abstraite, l'autre (celle de Külpe, inspirée d'Ernst Mach et de Richard Avenarius) visant l'unification de la science grâce à l'úsage généralisé des conceptualisations les plus abstraites possibles (ce qui entraînait la réduction de la psychólogie à la physiologie). La controverse s'amplifia, impliquant entre autres Dilthey, pour qui la psychologie était une science de l'esprit, et les «positivistes » Hermann Ebbinghaus et Edward B. Titchener.

Selon Wilhelm Dilthey (1833-1911); Comte et, après lui, Mill, Littré et Spencer n'avaient pas su troviver ce qui liait entre elles les sciences humaines. Leur activité systémátique ne s'était pas accompagnée du sentiment de la réalité historique. Or, seule l'histoire permettait d'appréhender le processus par lequel l'homme avait pris conscience de lui-même en tant qu'être différent de la nature; c'est cette conscience, selon Dilthey (1988, 1992), qui fonde l'autonomie des Geisteswissenschafien, dont le but est de 
comprendre le monde historique dans sa totalité. Dans la mesure où elle montre la réalité historique et sociale à l'état le plus simple, la psychologie est la base d'une telle compréhension, et donc la première et la plus élémentaire des sciences de l'esprit (voir aussi Tillich, 1981).

Le Cercle de Vienne attaquera Dilthey de front. Les tenants de l'empirisme logique verront dans la place de la psychologie parmi les sciences un des problèmes capitaux de l'épistémologie et de la métaphysique. Mais ils estimeront que toutes les propositions de la psychologie sont traduisibles, voire réductibles, au langage "physicaliste». Le langage de la science étant unitaire, les distinctions entre disciplines ne sauraient relever que d'aspects pratiques et de contenus particuliers (Hempel, 1935; Schlick, 1935).

Les tentatives taxinomiques si courantes à l'époque où la psychologie établissait ses assises intellectuelles et institutionnelles ne sont plus à la mode. Néanmoins, depuis les années 1930, le fond du débat opposant les sciences de la nature aux sciences de l'esprit n'est pas vraiment disparu. Il persiste, souvent implicitement, au point qu'il faut se demander si la question de la place de la psychologie dans l'ordre des sciences ne serait une interrogation irrévocablement constitutive de la discipline plutôt qu'un véritable problème à résoudre.

\section{LE CERCLE PIAGÉTHEN}

Toute l'œuvre de Jean Piaget (1896-1980) s'accompagne d'une réflexion sur la classification des sciences. Ses premières idées sur la question se trouvent dans son roman autobiographique, Recherche, de 1918. Il s'agit d'un cercle (d'après Piaget, $1918,1^{\text {re }}$ partie, chap. 8) :

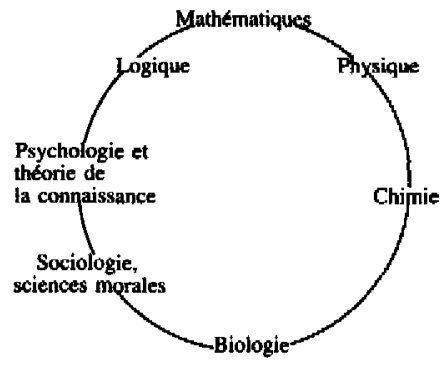

Le critère qui guide la construction de ce cercle n'est pas une quelconque relation génétique ou logique, mais le degré de dogmatisme des professionnels de chaque science. Le héros de Recherche critique toute la 
culture européenne. Le cercle des sciences représente alors le reproche de dogmatisme qu'il adresse aux scientifiques, en particulier aux biologistes de son époque. L'ensemble des sciences, dit-il, peut être conçu comme un cercle continu présentant deux pôles, l'un où le dogmatisme passe par son apogée (la biologie), l'autre par son minimum (les mathématiques) (ibid., p. 59). La mentalité dogmatique diminue à mesure que l'on va de la biologie à la mathématique, dans quelque sens que ce soit.

Piaget revient au sujet de la classification des sciences en 1929, lors de sa leçon inaugurale à la chaire d'histoire de la pensée scientifique de l'université de Genève. L'évolution des sciences lui semble procéder selon deux directions distinctes, la mathématique et la biologique. Dans la mesure où l'histoire des sciences est une réduction progressive de la réalité aux mathématiques, il s'agit de savoir comment et pourquoi les mathématiques, dont la vérité ne dépend pas de l'expérience, sont si bien adaptées à la connaissance du réel. La pensée s'explique selon Piaget par les « lois de l'organisation biologique »; dès lors, « le problème des rapports entre la pensée et les choses " apparaît comme « un cas particulier des relations entre l'organisme et le milieu "; et " les mathématiques et la biologie forment un cercle " (Piaget, 1929, p. 156, 159). Mais ce cercle, dit Piaget, n'est pas « absolu »; il ne se fermera jamais parce que les sciences ne sont jamais achevées. Il se transforme donc en une spirale où la pensée et le réel se créent mutuellement.

En 1950, Piaget reprend l'image du cercle et l'idée des deux directions de la pensée scientifique dans les conclusions de son Introduction à l'épistémologie génétique:

« Toute connaissance consistant en un rapport indissociable entre le sujet et l'objet, tel que l'objet soit seulement connu à travers son assimilation à l'activité du sujet et que le sujet se connaisse en retour lui-même par le seul intermédiaire de ses propres actions, c'est-à-dire de ses accommodations à l'objet, il n'est pas surprenant que ce cercle fondamental, inhérent à l'acte même de connaître, se retrouve dans l'ensemble des connaissances que constitue le système total de la pensée scientifique »(Piaget, 1950, p. 278).

Le savoir s'oriente dans une direction mathématique (où l'objet est "réduit " au sujet qui l'assimile à des cadres logico-mathématiques) et dans une direction biologique (où le sujet est « réduit » à l'objet au moyen de méthodes physico-chimiques).

Dans les réflexions ultérieures de Piaget sur l'ordre des sciences, la circularité des sciences devient un "cercle épistémologique fondamental " relevant de la « situation dialectique » du sujet et de l'objet. Les sciences sont logico-mathématiques, physiques, biologiques ou psycho-sociologiques. Chacune génère quatre domaines : le matériel, le conceptuel, l'épis- 
témologique interne et l'épistémologique dérivé; ce dernier englobe les conséquences épistémologiques des résultats scientifiques ${ }^{14}$ :

\begin{tabular}{l|l|l|l|l}
\hline Domaines $\rightarrow$ & A. Matériel & B. Conceptuel & C. Epistémologique interne & D. Epistémologique dérivé \\
\hline Sciences $\downarrow$ & & & & \\
\hline I. Logico-mathématiques & & & & \\
\hline II. Physiques & & & & \\
\hline III. Biologiques & & & & \\
\hline IV. Psychr-saciologiques & & & & \\
\hline
\end{tabular}

Pour Piaget, le cercle se dessine entre les domaines matériel et " épistémologique dérivé " de la psychologie et des mathématiques ; comme en 1929, il reflète l'interaction du sujet et de l'objet, ce qui met en relief la primauté épistémologique de la psychologie.

Piaget $(1966$, p. 253$)$ dit même «éprouver quelque fierté quant à la position clef qu'occupe la psychologie dans le système des sciences $»$. D'une part, explique-t-il, la psychologie dépend des autres sciences, puisqu'elle considère que la vie mentale est le produit des facteurs qu'elles étudient; mais d'autre part, aucune de ces sciences n'est possible sans une « coordination logico-mathématique » qui, loin d'être innée, se construit à travers les activités de l'organisme sưr les objets - activités dont la psychologie seule étudie le développement.

La place que Piaget attribue avec fierté à la psychologie est typique des classifications qui reposent suf un présupposé psychologiste. Lorsque l'épistémologie qui régit l'agencement des sciences prétend ramener toutes les formes de savoir à des processus psychologiques ou y trouver leurs fondements altimes, il est inévitable qu'elle fasse de la psychologie une science des sciences. Ceci a êté historiquement déterminant vers la fin du XvIII $^{e}$ siècle, à un moment où la psychologie trouve dans le discours encyclopédique qui lui donne un rang supérieur un moyen de se légitimer, de se construire en tant que domaine autonome, et finalement de côtoyer, lorsqu'elle quitte cette hauteur factice, non pas le spiritisme ou la chiromancie, mais les sciences reconnues comme telles. En bref, comme le souligne le refus initial du mot psychologie par ceux qui voulaient réduire la logique et la philosophie à l'investigation empirique de l'origine des idées, la psychologie aurait gagné sa place parmi les sciences grâce à une attitude psychologiste.

14. D'après $\propto$ Le système cyclique des sciences $», 2^{c}$ partie de « Le système et la classification des sciences \$, in PiaGET, éd., 1967. 
L'exemple extrême de ce processus est l'absorption de la logique par la psychologie au xix ${ }^{e}$ siècle : elle fut cruciale pour la formation du champ de la psychologie; et lorsque Edmund Husserl (1859-1938) s'est dressé contre le psychologisme, il le fit en voyant bien que, si la délimitation conceptuelle exacte du domaine d'une science ne détermine pas en principe son élaboration et sa fécondité, elle peut avoir des répercussions importantes sur la marche de la science elle-même (Husserl, 1990, Introduction, § 2).

Malgré la diversité des doctrines qui les fondent, tous les systèmes que nous avons examinés considèrent la psychologie comme la transition ou le lien entre les sciences physiques et biologiques d'une part, et d'autre part les sciences historiques et symboliques. Ceci est indépendant du rang hiérarchique qu'ils attribuent à la psychologie et des critères (méthodes, objets ou concepts de base) qu'ils emploient pour la classer. Dotée d'une identité hybride et d'une problématique frontière, la psychologie reste, tout au long du XIx ${ }^{e}$ siècle et jusqu'au moins les années 1930, le domaine le plus controversé de l'épistémologie et de la méthodologie des sciences. Réciproquement, elle est la discipline dont la place dans l'ordre des connaissances est la plus discutée parmi ceux qui la pratiquent. L'enjeu était considérable : il ne s'agissait pas de réfléchir à la systématique d'une science constituée, mais de constituer une science en se servant de la systématique.

Tout cela appartient encore au présent. Le radius reflexus de la métaphore optique de Bacon (1991, p. 138) - le rayon réfléchi par lequel l'homme se considère - conduit le regard à la fois vers le corps et vers ce qui, malgré l'emprise du discours neuronal, semble perpétuellement s'y dérober. Les meilleurs dictionnaires de la psychologie, anciens et récents, témoignent de la diversité des identités et des projets fondamentaux de cette science, ainsi que de l'incertitude des limites qui en découle. La psychologie n'a rien à y perdre. Vivant encore de ses origines lointaines, si elle tente parfois de n'être qu'empsychologia, c'est apparemment en sachant qu'elle ne peut cesser d'être psychologia sans s'aliéner à elle-même.

Fernando VIDAL, Faculté de psychologie et des sciences de l'éducation,

Université de Genève, CH-1227 Carouge-GE (1994). 


\section{BIBLIOGRAPHIE}

Autant que possible, nous faisons référence aux plus récentes éditions françaises, en indiquant la date de la première édition en langue originale.

ALSTED (Johann Heinrich), 1649, $1^{\text {re }}$ éd. 1630, Scientiarum Omnium Encyclopaedia, 4 t. en 2 vol., Lugduni, sumptibus J. A. Huguetan filii \& M. A. Ravaud.

Ampère (André-Marie), 1834/1843, Essai sur la philosophie des sciences, ou exposition analytique d'une classification naturelle de toutes les connaissances humaines, 2 vol., Paris, Bachelier.

Bacon (Francis), 1842, $1^{\text {re }}$ éd. 1623, De la dignité et de l'accroissement des sciences, in Euvres, trad. revue [de celle d'A. Lasalle] par F. RiauX, Paris, Charpentier, vol. 1.

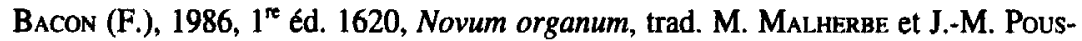
SEUR, Paris, Presses universitaires de France.

BACon (F.), 1991, $1^{\text {re }}$ éd. 1605, Du progrès et de la promotion des savoirs, trad. M. Le Defuf, Paris, Gallimard.

Bain (Alexander), 1894, $1^{\text {re }}$ éd. 1870, Logique déductive et inductive, vol. 1 : Déduction, trad. G. Compayre, Paris, Alcan.

Bentham (Jeremy), 1962, Works, éd. J. Bowring, New York, Russell and Russell, éd. de 1838-1843, vol. 8.

Braun (Claude M. J.) et Baribeau (Jacinthe M. C.), 1984, « The Classification of Psychology among the Sciences from Francis Bacon to Boniface Kedrov », Journal of Mind and Behavior, 5, p. 245-260.

Bréhier (Émile), 1986 (éd. revue, 1964), Histoire de la philosophie, Paris, Presses universitaires de France, vol. 3.

Bringmann (Wolfgang) et Tweney (Ryan D.), éd., 1980, Wundt Studies, Toronto, Hogrefe.

Brunet (Jacques-Charles), 1865 ( $5^{\mathrm{e}}$ éd.), $1^{\text {trt }}$ éd. 1810, Manuel du libraire et de l'amateur de livres, Paris, Firmin Didot, t. 6.

Coluson (Robert), 1966, Encyclopaedias : Their History throughout the Ages, New York, Hafner.

Condillac (Étienne Bonnot de), 1947-1951, $1^{\text {re }}$ éd. 1775, « Des progrès de l'art de raisonner ", Cours d'étude pour l'instruction du Prince de Parme, in G. LE Roy, éd., Euvres philosophiques de Condillac, Paris, Presses universitaires de France, vol. 2.

Cournor (Antoine-Augustin), 1975, $1^{\text {re }}$ éd. 1851, Essai sur les fondements de nos connaissances et sur les caractères de la critique philosophique, Paris, Vrin. 
Cournot (A.-A.), 1986, $1^{\text {re }}$ éd. 1875, Materialisme, vitalisme, rationalisme. Essai sur l'emploi des données de la science en philosophie, Paris, Vrin.

Couturat (Louis), 1966, $1^{\text {re }}$ éd. 1903, Opuscules et fragments inédits de Leibniz, Hildesheim, Olms.

Danziger (Kurt), 1979, "The Positivist Repudiation of Wundt ", Journal of the History of the Behavioral Sciences, 15, p. 205-230.

Dessolr (Max), 1964, $1^{\mathrm{r}}$ éd. 1902, Geschichte der neueren deutschen Psychologie, Amsterdam, E. J. Bonset.

Destutt de Tracy (Antoine-Louis-Claude), 1992, Mémoire sur la faculté de penser [...] et autres textes, Paris, Fayard.

DiltheY (Wilhelm), 1992, $1^{\text {Te }}$ ed. 1883, Critique de la raison historique; Introduction aux sciences de l'esprit et autres textes, préf. et trad. de S. Mesure, Paris, Cerf.

DiltheY (W.), 1988, $1^{\text {re }}$ ed. 1910, L'Édification du monde historique dans les sciences de l'esprit, trad. S. Mesure, Paris, Cerf.

Du Pleix (Scipion), 1990, $1^{\text {re }}$ éd. 1603, La Physique ou Science des choses naturelles, ed. de 1640, Paris, Fayard.

Du Pleix (S.), 1992, $1^{\text {re }}$ éd. 1610, La Métaphysique ou Science des choses surnaturelles, éd. de 1640, Paris, Fayard.

Engens (Friedrich), 1968, Dialectique de la nature [rédigé en 1875-1882], trad. E. BotTigell, Paris, Éditions sociales.

Goblor (Edmond), 1898, Essai sur la classification des sciences, Paris, Alcan.

Hartshorne (Charles) et Weiss (Paul), éd., 1960, $1^{\mathrm{rt}}$ éd. 1931, Collected Papers of Charles Sanders Peirce, Cambridge, Mass., Harvard University Press, vol, 1-2 en un tome.

Hegrel (Georg Wilhelm Friedrich), 1952, $1^{\mathrm{t}}$ éd. 1817, Précis de l'Encyclopédie des sciences philosophiques, trad. J. GIBELIN, Paris, Vrin.

Hempel (Carl G.), 1935, « Analyse logique de la psychologie ", Revue de synthèse, $\mathrm{X}, 1$, avril, p. 27-42.

HobBes (Thomas), 1971, 1" éd. 1651, Léviathan. Traité de la matière, de la forme et du pouvoir de la république ecclésiastique et civile, trad. F. TRLAUD, Paris, Sirey.

Hume (David), 1973, $1^{\text {re }}$ éd. 1739, Traité de la nature humaine : essai pour introduire la méthode expérimentale dans les sujets moraux, trad. A. LeRoY, 2 vol., Paris, Aubier.

HuSSERL (Edmund), 1990, $1^{\text {re }}$ éd. 1900, Recherches logiques, vol. 1 : Prolégomènes à la logique pure, trad. de la $2^{c}$ éd. 1913, H. ELIE, A. L. KelKEL et R. Scherer, Paris, Presses universitaires de France.

KÉDRov (Boniface), 1977-1980, La Classification des sciences, vol. 1 : Engels et ses prédécesseurs, vol. 2 : De Lénine à nos jours, trad. J. LemaGnen, Moscou, Ed. du Progrès.

Laubier (Jean), éd., 1974, Auguste Comte. Philosophie des sciences. Textes choisis, Paris, Presses universitaires de France.

Leary (David), 1979, «Wundt and After : Psychology's Shifting Relations with the Natural Sciences, Social Sciences, and Philosophy ", Journal of the History of the Behavioral Sciences, 15, p. 231-241. 
Lévy-Bruhl (Lucien), 1900, La Philosophie d'Auguste Comte, Paris, Alcan.

Litrré (Émile), 1876, La Science au point de vue philosophique, Paris, Didier.

LockE (John), 1989, $1^{\text {re }}$ éd. 1690, Essai philosophique concernant l'entendement humain, trad. P. Coste, éd. de 1755, Paris, Vrin.

McRae (Robert F.), 1961, The Problem of the Unity of Knowledge : Bacon to Kant, Toronto, University of Toronto Press.

Mitl (John Stuart), 1897, $1^{\text {re }}$ éd. 1843, La Logique des sciences morales [Système de logique déductive et inductive, liv. 6], trad. G. BELoT, Paris, Delagrave.

Mill (J. S.), 1898, $1^{\text {re } e ́ d . ~ 1865, ~ A u g u s t e ~ C o m t e ~ e t ~ l e ~ p o s i t i v i s m e, ~ t r a d . ~ G . ~ C l e m E N-~}$ CEAU, Paris, Alcan.

Mounasseb (Jamal), 1971-1972, $1^{\text {re }}$ éd. 1953, La Classification des sciences. Étude historique, sociologique et philosophique, vol. 1: Les Philosophes grecs et arabes, vol. 3: L'Epoque contemporaine [il n'y a pas de vol.2], Beyrouth, Imprimerie catholique.

Naville (Adrien), 1887, « De la classification des sciences. Étude logique », La critique philosophique, 3 (2), p. 321-342, 401-415.

NAville (A.), 1920, Classification des sciences. Les idées maîtresses des sciences et leurs rapports, Paris, Alcan.

Novalis, 1966, L'Encyclopédie. Notes et fragments [rédigé 1795-1800], trad. M. de Gandillac, Paris, Minuit.

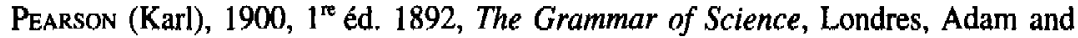
Charles Black.

Plaget (Jean), 1918, Recherche, Lausanne, La Concorde.

Piaget (J.), 1929, "Les deux directions de la pensée scientifique ", Archives des sciences physiques et naturelles, année 134, $5^{\mathrm{C}}$ période, vol. 11, p. 145-162.

Piaget (J.), 1950, Introduction à l'épistémologie génétique, vol. 3 : La Pensée biologique, la pensée psychologique et la pensée sociologique, Paris, Presses universitaires de France.

Piaget (J.), 1966, « La psychologie, les relations interdisciplinaires et le système des sciences », Bulletin de psychologie, 20, p. 242-254.

Piaget (J.), éd., 1967, Logique et connaissance scientifique, Paris, Gallimard « Encyclopédie de la Pléiade ».

Renouvier (Charles), 1912, $1^{\text {te }}$ éd. 1859, « De la certitude des sciences et de leur classification rationnelle $»$, in Traité de psychologie rationnelle d'après les principes du criticisme. Essais de critique générale. Deuxième essai, Paris, A. Colin, vol. 2.

Richardson (Emest Cushing), 1964, $1^{\text {re }}$ éd. 1901, Classification Theoretical and Practical, Hamden (Connecticut), Shoe String Press.

Saint-Simon (Claude-Henri, comte de), 1973, Euvres choisies, éd. de 1859, Hildesheim, G. Olms.

Savonarole (Girolamo), 1982-1988, Scritti filosofici, éd. G. GarfaGninI et E. Garin, 2 vol., Rome, A. Belardetti.

Savonarole (G.), 1989, $1^{\text {re }}$ éd. 1491, La Fonction de la poésie, trad. Bruno PinCHARD, Lausanne, L'Âge d'homme.

SCHLICK (Moritz), 1935, « De la relation entre les notions psychologiques et les notions physiques ", Revue de synthèse, X, 1, avril, p. 5-26. 
SPENCER (Herbert), 1854, «The Genesis of Science », in Essays Scientific, Political, and Speculative, New York, Appleton, 1907, vol. 2.

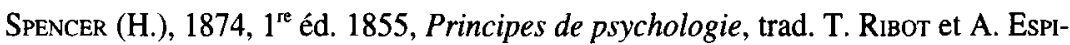
NAS, 2 vol., Paris, G. Baillère, vol. 1.

Spencer (H.), 1894, $1^{\text {te }}$ éd. 1862, Les Premiers principes, trad. M. E. Cazelles, Paris, F. Alcan.

SPEnCER (H.), 1909, La Classification des sciences, trad. F. RÉTHORÉ, éd. de 1871, Paris, Alcan.

Speziali (Pierre), 1973, « Classification of the Sciences ", in P. P. WIENER, éd., Dictionary of the History of Ideas, New York, Charles Scribner's Sons, vol. 1.

TIllich (Paul), 1981, $1^{\text {re }}$ éd. 1923, The System of the Sciences according to Objects and Methods, trad. P. WIEBE, Londres/Toronto, Associated University Presses.

Vidal (Fernando), 1993, "Psychology in the 18th Century: A View from Encyclopaedias », History of the Human Sciences, 6, p. 89-119.

Whewell (William), 1858, Novum organon renovatum, Londres, John W. Parker and Son.

WundT (Wilhelm), 1889, «Ueber die Eintheilung der Wissenschaften ", Philosophische Studien, 5, p. 1-55.

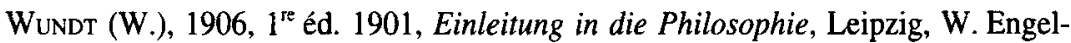
mann.

WundT (W.), 1907, Logik, vol. 2 : Logik der exakten Wissenschaften, $3^{\mathrm{e}}$ éd., Stuttgart, F. Enke. 